Japan. J. Math.

Vol. 19, No. 2, 1994

\title{
On the geography of ample and spanned vector bundles on algebraic surfaces
}

\author{
By E. BALlico \\ (Received September 4, 1992)
}

In recent years there were several papers dealing (from different point of view) with ample vector bundles (often assumed to be spanned by global sections) on a smooth projective surface, $X$. In particular there appeared (see e.g. [B1], [B2], $[\mathrm{F}],[\mathrm{Ru}]$ ) classifications of all such pairs (surface, bundle) when the bundle, $E$, has "low" numerical invariants and inequalities on the possible numerical invariants $\left(c_{1}(E)^{2}, c_{2}(E)\right.$ and the sectional genus $g(\operatorname{det}(E))$ of $\left.\operatorname{det}(E)\right)$ (see e.g. [F], [B2], [LR]). Often these inequalities were "sharp" in the sense that the authors gave borderline examples showing that, with the assumptions made in the corresponding statement, nothing more could be proved. However, the examples were always very "special". It seems to us that to obtain much more one need to discuss separately the problem on $(X, E)$ for geometrically very different $X$ (e.g. with different Kodaira dimension $\kappa(X))$; indeed to obtain "good" bounds on, say, $g(\operatorname{det}(E))$ in terms of $\chi\left(\mathbf{O}_{X}\right)$ it is convenient to discuss separately surfaces with $\chi\left(\mathbf{O}_{X}\right)<0$ and with $\chi\left(\mathbf{O}_{X}\right) \geq 0$. This is exactly the subject of $\S 5$ of this paper, the main results (theorem 5.4.2 and Remark 5.4.3) being obtained when $X$ has general type.

We stress another phenomenon: there are big jumps in the possible Chern numbers of such bundles (for rank 2 see theorem 7.1 in $\S 7$ ).

We stress that much stronger results can be obtained if one considers separately each numerical invariant $\left(c_{1}^{2}, c_{2}, g(\operatorname{det}(E)), h^{0}(X, E)\right.$ ) (or, better, a good combination of them, as in $[\mathrm{F}]$ and $[\mathrm{B} 2])$, since in different geometric situations it is often possible to obtain more for one of them and then use the informations gained on that invariant (e.g. to exclude doubtful cases in classification lists). Here we only remark that the case " $h^{0}(X, E) \leq 2 \operatorname{rank}(E)$ " and the case " $h^{0}(X, E)>2 \operatorname{rank}(E)$ " seems to be geometrically and numerically very different (at least for stable bundles).

In $\S 1$ we collect several technical definitions and a few elementary remarks. The subject of sections 2,3 , and 4 is the construction of suitable rank 2 bundles and the checking that they are, for instance, ample and spanned. In sections 2 and 3 we start with a suitable bundle $E$ on $X$. In $\S 2$ we construct from $E$ other bundles 
on $X$ with the same positivity properties and covering a large range for the Chern classes; the construction is made using elementary transformations (in the sense of [Ma]). In $\S 3$ we consider the blowing up $f: S \rightarrow X$ of $X$ at a point $P$ and show (using elementary transformations with support $f^{-1}(P)$ ) when (assuming only the existence of $E$ ), there is a "good" bundle, $F$ on $S$ with given $F \mid f^{-1}(P)$. In $\S 4$ we make a construction of ample and/or spanned stable rank two vector bundles: see theorem 4.7. In $\S 6$ we consider (of course only in positive characteristic) bundles on uniruled surfaces of general type.

The author was partially supported by MURST and GNSAGA and CNR (Italy).

$\S 1$. Here we collect a long list of definitions which later will be useful to have stronger statements and/or easier proofs. We will refer to any of them as a "positivity condition". Some of them seem to have some intrinsic interest. In this paper we work over an algebraically closed field $\mathbf{K}$; in some of the main results we will assume $\operatorname{char}(\mathbf{K})=0$.

Definition 1.1. A vector bundle $E$ on a projective variety $V$ is called cample if for every integral curve $T \subset V$, the bundle $E \mid T$ is ample.

Definition 1.2. A vector bundle $E$ on an integral projective variety $V$ is called almost c-ample if there is an algebraic subset $B$ of $V$ with $B \neq V$ and such that the bundle $E \mid T$ is ample for every integral curve $T \subset V$ with $T$ not contained in $B$.

Definition 1.3. A vector bundle $E$ on an integral projective variety $V$ is called generically c-ample if there is an algebraic subset $D$ of $V$ with $\operatorname{codim}(D)>1$ and such that the bundle $E \mid T$ is ample for every integral curve $T \subset V$ with $T \cap D=$ $\emptyset$.

Definition 1.4. A vector bundle $E$ on a smooth curve $D$ is called qc-ample if all its non zero quotients (including $E$ itself) have positive degree. Using this notion we leave to the reader to write down explicitly definitions $1.1,1.2$ and 1.3 with qc-ampleness instead of c-ampleness.

REMARK 1.5. If $\operatorname{char}(\mathbf{K})=0$ a $q c$-ample bundle on a smooth curve is ample ([H2], prop. 2.3); however, in general this is not true when $\operatorname{char}(\mathbf{K})>0([\mathrm{H} 2])$.

REMARK 1.6. In any characteristic a qc-ample and spanned bundle is ample ([Gi], prop. 2.1).

REMARK 1.7. Assume $p:=\operatorname{char}(\mathbf{K})>0$ and let $E$ be a rank $r$ vector bundle on a smooth curve $D$ of genus $q$. If $E$ is $q c$-ample and $q=1$, then $E$ is ample (e.g. [O], or [H2], prop. 1.2). For higher $q$ and $r=2$ this is true if $\operatorname{deg}(E)>(2 q-2) / p$ 
([H1])

Definition 1.8. A vector bundle $F$ on a variety $V$ is called c-spanned if for every integral curve $T \subset V$ the bundle $F \mid T$ is spanned.

REMARK 1.9. The classification of vector bundles on $\mathbf{P}^{1}$ gives that for every rank $r$ qc-ample bundle $E$ on $\mathbf{P}^{1}$ we have $\operatorname{deg}(E) \geq r$ with equality if and only if $E$ is the direct sum of $r$ line bundles of degree 1 .

$\S 2$. The following construction (called elementary transformation ([Ma $]$ )) will be used in this section and the next one to construct bundles with positivity properties and many Chern classes.

Construction 2.1: Let $S$ be a smooth projective surface and let $D \subset S$ be a reduced curve. Fix a vector bundle $F$ on $S, \operatorname{rank}(F)=r$. Let $\mathbf{c}$ be the conormal bundle of $D$ in $S$. Let $U$ be a vector bundle over $D$ with $\operatorname{rank}(U) \leq r$, and fix a surjective map $t: F \mid D \rightarrow U$. Let $F^{\prime}$ be the kernel of the induced surjective map $F \rightarrow F \mid D \rightarrow U$; hence we have

$$
0 \rightarrow F^{\prime} \rightarrow F \rightarrow U \rightarrow 0
$$

$F^{\prime}$ is called the elementary transformation of $F$ along $t$. Note that if $U$ is a line bundle, then in the Chow ring of $S$ we have $c_{1}(U)=D$ and $c_{2}(U)=D^{2}-\operatorname{deg}(U)$ (hence $c_{1}\left(F^{\prime}\right)=c_{1}(F)-D$ and $c_{2}\left(F^{\prime}\right)=c_{2}(F)+u-D \cdot c_{1}(F)$ ). For the elementary properties of this notion (and the motivations for introducing it) see $[\mathrm{Ma}]$. By the exact sequence:

$$
0 \rightarrow \mathbf{O}(-D) \rightarrow \mathbf{O} \rightarrow \mathbf{O}_{D} \rightarrow 0 .
$$

we get that $\operatorname{Tor}_{\mathbf{O}}^{1}\left(\mathbf{O}_{D}, \mathbf{O}_{D}\right)$ is a line bundle on $D$ isomorphic to $c$. Thus we get the exact sequence on $D$ :

$$
0 \rightarrow U(c) \rightarrow F^{\prime} \mid D \rightarrow \operatorname{Ker}(t) \rightarrow 0
$$

The surjection $s: F^{\prime} \mid D \rightarrow \operatorname{Ker}(t)$ in (3) induces an elementary transformation of $F^{\prime}$ with respect to $s$. It is easy to check that the corresponding bundle is isomorphic to $F(-D)$, i.e. we have the exact sequence:

$$
0 \rightarrow F(-D) \rightarrow F^{\prime} \rightarrow \operatorname{Ker}(t) \rightarrow 0
$$

If $T$ is an integral curve with $\operatorname{dim}(T \cap D) \leq 0$, then restricting (4) to $T$ we see that $F^{\prime} \mid T$ contains $(F \mid T) \otimes \mathbf{O}_{T}(D)$ as a subsheaf; this is very useful to check the c-ampleness or c-spannedness of a twist of $F^{\prime}$.

In [B4] it was described in detail what may happen when $D$ is a smooth rational curve on $S$. In $\S 3$ we will be interested in the case in which $D$ is an exceptional 
curve. In this section we will use the following data to obtain on any surface $X$ from a "positive" bundle $E$ many other "positive" bundles.

(2.2): Fix the surface $X$, a rank 2 vector bundle $E$ on $X, M \in \operatorname{Pic}(X)$, two reduced curves $\mathrm{A}, \mathrm{B}$ in $[\mathrm{M}]$ with $\operatorname{dim}(A \cap B)=0$, and integers $u, v$ with the following properties. First, there is a surjection $s: E \mid A \rightarrow \mathbf{u}$ (u line bundle of degree $u$ on A); $s$ induces the following elementary transformation:

$$
0 \rightarrow E^{\prime} \rightarrow E \rightarrow \mathbf{u} \rightarrow 0
$$

Then there is a surjection $s^{\prime}: E^{\prime} \mid B \rightarrow \mathbf{v}$ with target a line bundle of degree $v$ on $\mathrm{B} ; s^{\prime}$ induces the following elementary transformation:

$$
0 \rightarrow E^{\prime \prime} \rightarrow E^{\prime} \rightarrow \mathbf{v} \rightarrow 0
$$

Assume $M$ spanned. If $E$ has any of the positivity conditions considered in this paper, $E \otimes M$ has the same properties; we will show that for suitable $u$ and $v$ this construction gives bundles (of the form $E^{\prime \prime} \otimes M^{\otimes 2}$ ) with the same positivity conditions, same $c_{1}$ and many possible $c_{2}$ near to $c_{2}(E \otimes M)$. Set $E_{1}:=E^{\prime \prime} \otimes M^{\otimes 2}$. With these data we have:

$$
c_{2}\left(E_{1}\right)=c_{2}(E \otimes M)+u+v-M \cdot c_{1}(E)
$$

To check positivity and give an explicit statement we will consider a case which is in principle the worst possible one (i.e. $v=u$ and $u$ large).

TheOREM 2.3. Assume $E$ c-spanned, $M$ spanned and $M^{2}>0 ;$ let $g(M)$ be the sectional genus of $M$. Then we may do the construction 2.2 for the integers $u=M \cdot c_{1}(E)+h, \nu=M \cdot c_{1}(E)$ and find $E_{1} c$-spanned if either $h=0$ or $0<$ $-2 h \leq\left(M \cdot c_{1}(E)-5 g(M)-2\right)$ and $A$ is smooth. Furthermore, if $E$ is c-ample (resp. spanned, resp. ample), then we will obtain a c-ample (resp. spanned, resp. ample) bundle $E_{1}$. Fix integers $h, x$ with $h>0, x \geq 0,2 h+M \cdot c_{1}(E) \geq 5 g(M)-2$ and $x M^{2} \geq h+2 g(M)$; then we can make the construction 2.2 obtaining c-spannedness (or c-ampleness, or ampleness or spannedness) for $E_{1} \otimes M^{\otimes x}$.

Proof. First we assume $h=0$. Since $E \mid A$ is spanned, we have a nowhere zero section of $E \mid A$ inducing a surjection, $s$, with image $\mathbf{u}:=c_{1}(E) \mid A$. We obtain $E^{\prime}$. Note that the restriction of (5) to $B$ gives the following exact sequence:

$$
0 \rightarrow E^{\prime}|B \rightarrow E| B \rightarrow \mathbf{O}_{A \cap B} \rightarrow 0
$$

Set $v:=\operatorname{det}(E) \mid B$. Taking a general nowhere vanishing section of $E \mid B$ we obtain a surjection $s^{\prime \prime}: E \mid B \rightarrow v$; hence restricting (5) to $B$ a morphism $s^{\prime}: E^{\prime} \mid B \rightarrow v$. We claim that we may obtain $s^{\prime}$ surjective; indeed we may fix $B$ and vary independently 
$A, s$ and $s^{\prime \prime}$; by (8) a general choice of $A, s$ and $s^{\prime \prime}$ proves the claim. For the campleness of $E_{1}$ we use (4),(5),(6) and (8) to check separately the ampleness of $E_{1} \mid T$ when $T$ is either $A$ or $B$ or a curve different from $A$ and from $B$. For the spannedness and ampleness statement, use (4).

Now assume $h<0$. We make the same construction on $B$ to pass $E^{\prime}$ to $E^{\prime \prime}$; in the construction involving $A$ we use any $\mathbf{u}$ with the correct degree; we claim (and prove below) the existence of a surjection with target $\mathbf{u}$ with the correct degree. Assuming the claim, we get the bundle $E_{1}$. To check the c-spannedness, spannedness, ampleness or the c-ampleness of $E_{1}$ the only difference arises for the curve $A$; the assumptions on $t$ and $M$ are exactly made to handle this case. To prove the claim we use that by a theorem of $C$. Segre and Nagata (see $[N]$ ) there is an exact sequence:

$$
o \rightarrow \alpha \rightarrow E \mid A \rightarrow \beta \rightarrow 0
$$

with $\alpha$ and $\beta$ line bundles on $A$, say of degree $a$ and $b$, and with $b-a \leq g(M)$. This inequality, the assumption on $h$ and (9) shows (since the involved line bundles have degree at least $2 g(M)+1$, hence in particular induce embeddings of $A$ ) that $E$ has a section vanishing at exactly $-h$ points.

Now assume $h>0$. The condition on $h$ is just enough to say from the inequality $b-a \leq g(M)$ and (9) that for every line bundle $R$ of degree $>-h$ the bundle $\left(E^{*} \mid A\right) \otimes R$ is an extension of two line bundles of degree at least $2 g(M)-1$, hence with no higher cohomology; this implies the existence of a morphism $E \mid A \rightarrow \mathbf{u}$ (for some $\mathbf{u}$ of degree $h+c_{1}(E) \cdot M$ ) which does not factor through a lower degree line bundle, i.e. that it is surjective. The condition on $x$ implies in the usual way the positivity assertions for $E_{1} \otimes M^{\otimes x}$.

§3. Let $f: S \rightarrow X$ be the blowing up of $X$ at a point $P$ and let $D$ be the exceptional divisor (hence in the construction 2.1 we have $\operatorname{deg}(\mathbf{c})=1$ ). Twisting (2) by $\mathbf{O}(-t D)$, we see that a line bundle of degree $t$ on $D$ has in th Chow ring of $S$ the class of $D$ as $c_{1}$ and the integer $-t-1$ as $c_{2}$. We fix a rank 2 vector bundle $E$ on $X$, a line bundle $M$ on $X$ and two integers $a, b$ with $a \geq b>0$. With these data we want to find a rank 2 vector bundle, $G$ on $S$ with $G \mid D$ of splitting type $(a, b)$, and with "positivity properties" depending on positivity properties of $E$ and $M$. Set $F:=f^{*}(E)$.

Case 3.1 If $a=b$ we take $G:=F(-a D)$. Hence $c_{1}(G)=f^{*}\left(c_{1}(E)\right)-2 a D$, $c_{1}(G)^{2}=c_{1}(E)^{2}-4 a^{2}$ and $c_{2}(G)=c_{2}(E)-a^{2}$.

Case 3.2 Assume $a>b$ and $a-b$ odd, say $a-b=2 m+1$. In the construction 2.1 of $\S 2$ we take $\operatorname{rank}(U)=1, \operatorname{deg}(U)=m$ and take as $t$ any surjection $t: F \rightarrow$ $F \mid D \rightarrow U$. Hence $F^{\prime}:=\operatorname{Ker}(t)$ has splitting type $(m+1,-m)$ on $D ;$ set $G: F^{\prime}(-(b+$ $m) D)$. Hence $c_{1}(G)=f^{*}\left(c_{1}(E)\right)-(2 b+2 m+1) D, c_{1}(G)^{2}=c_{1}(E)^{2}-(2 b+2 m+1)^{2}$ and $c_{2}(G)=c_{2}\left(F^{\prime}\right)-(b+m) c_{1}\left(F^{\prime}\right) \cdot D-(b+m)^{2}=c_{2}(E)+b+2 m-1-(b+m)^{2}$. 
Case 3.3 Assume $a>b$ and $a-b$ even, say $a-b=2 m$. First we take $U, t$ and $F^{\prime}$ as in the previous case. Then we take a degree $-m$ line bundle $U^{\prime}$ on $D$ and take a surjection (unique up to a constant) $v: F^{\prime} \rightarrow U$. Set $F^{\prime \prime}:=\operatorname{Ker}(v) ; F^{\prime \prime}$ has splitting type $(m+1,1-m)$ on $D$. Set $G:=F^{\prime \prime}((-b-m+1) D)$.

Assume that $E$ is c-ample and let $\Gamma \in|A-x D|$ be an integral cuve with $A \in f^{*}(\operatorname{Pic}(X))$ and $x \geq 0$; set $B:=f(\Gamma)$. Since $G \mid \Gamma$ is the tensor product of $f^{*}(E \mid B)$ by a line bundle of degree $x b \geq 0$, in case $3.1 G$ is c-ample (or qc-ample) if $E$ has the same property. We claim that the same is true in cases 3.2 and 3.3. Restricting (4) to $\Gamma$ we obtain the claim if $a-b$ is odd. Iterating the construction we obtain the claim if $a-b$ is even.

Now we consider the spannedness. We take $M \in \operatorname{Pic}(X)$ with $M$ spanned and such that $f^{*}(M)(-b D)$ in case 3.1 (resp. $f^{*}(M)(-(b+m+1) D)$ in case 3.2 and $f^{*}(M)(-(b+m+2) D)$ in case 3.3) is spanned. Then $G \otimes f^{*}(M)$ is spanned (e.g. use again (4) for cases 3.2 and 3.3). This has a tautological interpretation in terms of the higher order jets of $X$ at $P$. Of course, instead of just a point one can do simultaneously any number of blowing ups and then iterate.

REMARK 3.4. Fix an integer $j>0$. It is possible to find (starting with $E$ ) bundles on $S$ with good positivity properties and with prescribed restriction to a $j$ th neighborhood $(k+1) D$ of $D$ (for the numerology involved, [B4] may be helpful). We only stress that two bundles $G$ and $G^{\prime}$ with splitting type $(a, b)$ on $D$ (with $a \geq b$ ) are isomorphic in a formal neighborhood of $D$ if their restrictions to $(a-b) D$ are isomorphic (use standard exact sequences to lift any such isomorphism).

REMARK 3.5. It is possible (and conceptually easy, but the numerology is messy) to make the same construction for higher rank.

It is often very easy to pass from a bundle on $S$ with good positivity properties to a bundle on $X$ with the same related positivity properties.

$\S 4$. Let $X$ be a smooth projective surface; set $\mathbf{O}:=\mathbf{O}_{X}$. Fix an ample $H \in \operatorname{Pic}(X)$. Consider a rank 2 vector bundle $E$ on $X, E$ given by an extension:

$$
0 \rightarrow M \rightarrow E \otimes M \rightarrow I_{Z}(L+M) \rightarrow 0
$$

with $M \in \operatorname{Pic}(X), L=\operatorname{det}(E)$ and $Z$ a locally complete intersection zero dimensional subscheme of $X$; set $z:=\operatorname{length}(Z)$, i.e. set $z=c_{2}(E)$. Varying $Z$ and the extension (10), we obtain many different bundles $E$ and we want to give necessary or sufficient condition for these data and $M$ for the ampleness or spannedness of $E \otimes M$. In this section we are interested in particular in the (harder) case of stable $E$; according to ampleness or spannedness properties of $E \otimes M$ we have a stratification of the moduli scheme $M\left(c_{1}, c_{2} ; H\right)$ of stable vector bundles (with respect to $H$ ) on $X$ with given Chern classes $c_{1}$; recall that here we consider stability in 
the sense of Mumford-Takemoto, i.e. slope stability with respect to the polarization $H$. We will say that $E$ belongs to a good stratum if $Z$ is reduced and formed by "general" points of $X$ (here "general" may means "generic in the Grothendieck sense as a point in the $c_{2}$-symmetric product of $X$ " or "generic in Weil sense" or (when $\mathbf{K}$ is uncountable) "outside countably many proper suitable subvarieties of the $c_{2}$-symmetric product of $X$ " (although for numerical reasons and the cohomology of line bundles on $X$ we usually may mean with a little more of care "outside finitely many proper suitable subvarieties of the $c_{2}$-symmetric product of $X$ "). We do not impose that a bundle in a good stratum is $H$-stable (and $L \cdot H$ may be negative), but quite often (depending on $L$ and $c_{2}$ ) every bundle in a good stratum is $H$-stable.

REMARK 4.1. By Cayley-Bacharach there is a good stratum for $\left(L, c_{2}\right)$ if and only if $c_{2}>h^{0}(K \otimes L)$.

REMARK 4.2. By definition of good stratum and the countability of the set of all integral curves $C \subset X$ with $C^{2}<0$, we may assume $C \cap Z=\emptyset$ for all pairs $(C, Z)$ with $Z$ in (10) defining a bundle in a good stratum and $C^{2}<0$.

Remark 4.3. Fix an integral curve $T \subset X$ with $T^{2}>0$. Note that $h^{0}(T) \leq$ $1+h^{0}\left(T, \mathbf{O}_{T}(T)\right)$. Use Riemann-Roch. Thus if $T$ is smooth and rational, then $h^{0}(T) \leq T^{2}+2$, while if $P_{a}(T)>0$, then $h^{0}(T) \leq T^{2}+1$. If $\kappa(X) \geq 0$ and $h^{0}(T) \geq 3$, then the base locus, $B$, of $|T|$ has length $(B)<T^{2}$; hence (by Clifford theorem on $T$ ) we have $h^{0}(T) \leq 2+\left(T^{2}\right) / 2$; using this, one can have for free a better bound in the statements 4.6 and 4.7 below.

REMARK 4.4. By Hodge index theorem and 4.3 the condition on $T \cdot(L+M)$ in 4.5 below is satisfied for all integral $T$ with $T^{2}>0$ if $(L+M)^{2} \geq c_{2}+3$ and $L+M$ is, say, nef. Hence by 4.2 in the application (theorem 4.7), assuming the c-ampleness of $L+M$ and that $(L+M)^{2} \geq c_{2}+3$, only the curves with $T^{2}=0$ and $h^{0}(T)>1$ (hence $|T|$ base point free) may give troubles. However, a generic $Z$ intersect each fiber of the morphism $h_{|T|}$ induced by $|T|$ at most in a point; hence even this case gives no trouble.

Lemma 4.5. Assume $M$ ample and that for every integral curve $T \subset X$ we have $T \cdot(L+M) \geq \min \left(h^{0}(T), c_{2}+1\right)$. Then for every bundle $E$ in the good stratum for $\left(L, c_{2}\right)$, the bundle $E \otimes M$ is c-ample.

Proof. Fix an integral curve, $T$, and let $f: C \rightarrow T$ be its normalization. Restrict (10) to $T$; note that the induced map $M|T \rightarrow E \otimes M| T$ is injective (as a map of sheaves) because $M \mid T$ is torsion-free and $T$ has no embedded point. By definition of good stratum we have card $(Z \cap T) \leq \min \left(c_{2}, h^{0}(X, L \otimes M)\right)$ (here if $\operatorname{dim}(\operatorname{Pic}(X))>0$ we use semicontinuity to have this for all $L \otimes M)$. Hence it is easy to check that $f^{*}(E \otimes M \mid T)$ is an extension of a line bundle of degree>0 (hence 
ample) by $M \mid T$.

Proposition 4.6. Fix $M \in \operatorname{Pic}(X)$ and a rank 2 vector bundle $E$ in the good stratum for $\left(L, c_{2}\right)$. Assume $(L+M)^{2} \geq c_{2}+3$. Then $E$ is c-ample.

Proof. Just apply 4.3 and 4.5 .

Theorem 4.7. Fix $M \in \operatorname{Pic}(X)$ with $M$ spanned, $h^{1}(M)=0, L \otimes M$ spanned, $h^{0}(L \otimes M) \geq c_{2}+3$ and such that the morphism, $h:=h_{|L \otimes M|}$, induced by $H^{0}(L \otimes M)$ is birational. Then for every vector bundle $E$ in the good stratum for $\left(L, c_{2}\right)$, the bundle $E \otimes M$ is spanned.

Proof. By (10) it is sufficient to check that $\mathbf{I}_{Z} \otimes(L \otimes M)$ is spanned. By the birationality of $h$, for a general $P \in X, \mathbf{I}_{\{p\}} \otimes(L \otimes M)$ is spanned. Set $P_{1}:=P$; take $c_{2}-1$ general projection from points $x_{i}:=h\left(P_{i}\right)$ with $P_{i} \in X, 2 \leq i \leq c_{2}$; set $Z:=\left\{P_{i}\right\}_{i \geq 1}$; we see easily that $\mathbf{I}_{Z} \otimes(L \otimes M)$ is spanned and $h^{0}\left(\mathbf{I}_{Z} \otimes(L \otimes M)\right)=$ $h^{0}(L \otimes M)-c_{2}$. Hence for generic $Z$ the same is true. Alternatively, one could use that any symmetric product of $X$ is irreducible and a "monodromy" argument.

Note that for generic $Z$ the birationality of $h=h_{|L \otimes M|}$ is a necessary condition for the spannedness of $E$; for non generic $Z$ if $h$ is not birational we cannot have simultaneously the spannedness of $\mathbf{I}_{Z} \otimes(L \otimes M)$ and the equality " $h^{1}\left(\mathbf{I}_{Z} \otimes(L \otimes\right.$ $M))=h^{1}(L \otimes M)^{\prime \prime}$.

REMARK 4.8. The proof of 4.7 gives results for $k$-spannedness and $k$-very ampleness of $E$ in any "possible sense"; for all reasonable "possible sense", recall that a spanned vector bundle is ample if it is c-ample (remark 1.6).

$\S 5$. Let $X$ be a smooth projective surface and $E$ a rank $r$ bundle on $X$. In this section we will give lower bounds for the sectional genus $g(\operatorname{det}(E))$ of $\operatorname{det}(E)$, assuming that $E$ is "positive". We distinguish 4 cases according to the possible values of the Kodaira dimension $\kappa(X)$ of $X$. In this section (except in 5.1, i.e. when $\kappa(X)<0)$ we will assume $\operatorname{char}(\mathbf{K})=0$

(5.1) Assume $\kappa(X)<0$. We will assume $X \neq \mathbf{P}^{2}$. Let $f: X \rightarrow A$ be a ruling of $X$ with $A$ smooth curve of genus $q$.

LEMMA 5.1.1. Let $T$ be a general fiber of the ruling $f$.

(i) We have $\operatorname{deg}(L / T) \geq r$ if one of the following assumptions is satisfied:

(a1) E is ample;

(a2) $E$ is c-ample;

(a3) $E$ is almost c-ample;

(a4) E is generically c-ample;

(a5) $E$ is qc-ample. 
(ii) If $f$ is not minimal and $E$ is ample (or at least qc-ample) then $\operatorname{deg}(L / T) \geq 2 r$.

(iii) If $f$ is minimal and $E$ is ample (or at least qc-ample) and $\operatorname{deg}(L / T)=r$, then there is a section $H$ of $f$ and rank $r$ vector bundle $F$ on $A$ with $E \cong f^{*}(F)(H)$.

Proof. Just use Remark 1.9. The assertion (iii) is just an easy and well known consequence of a base change theorem.

From the Riemann-Hurwitz formula we obtain the following result:

Corollary 5.1.2. If $E$ is almost c-ample, $H^{0}(X, L)$ spans $L$ outside finitely many points and a general curve in $|L|$ is integral, then $g(L) \geq r q-r+1$. Furthermore, if $X$ is not a relatively minimal model, then $g(L) \geq 2 r q-2 r+1$. If $X$ is a relatively minimal model, then $g(L) \geq(r+1) q-r$ unless $E \cong f^{*}(F)(H)$ for a section $H$ of $f$ and a bundle $F$ on $A$.

(5.2) If $\kappa(X)=0$ (hence $q \leq 2, p_{g} \leq 1$ and $\chi\left(\mathbf{O}_{X}\right) \geq 0$ ) we do not have any lower bound for $g$ which does not follow from the classification of surfaces with low sectional genus. Of course, if $X$ is not minimal (say with $\tau:=-K^{2}$ ) we have $2 g-2=L^{2}+K \cdot L \geq L^{2}+r \tau$ by Remark 1.9 .

(5.3) Now assume $\kappa(X)=1$. Let $u: X \rightarrow A$ be the elliptic fibration; set $a:=P_{a}(A)$ (hence $a \leq q \leq a+1$ ([BPV])). Let $T$ be a general fiber of $u$. Set $s:=\operatorname{deg}(E \mid T)$. By Riemann-Hurwitz we have $g(L) \geq s a-a+1$. If $E$ is $c$-spanned and $c$-ample, then $s \geq 3$ and if we have equality, then all the fibers of $E$ are integral.

(5.4) Now we consider the case $\kappa(X)=2$. Let $X^{\prime}$ be the minimal model of $X$; set $c:=K_{X^{\prime}}^{2}$ and $\tau:=c-K^{2}$ (i.e. $X$ is obtained from $X^{\prime}$ with $\tau$ blowing ups).

REMARK 5.4.1. Since $K L \geq r \tau$ by 1.9 , we have $g \geq 1+(r \tau) / 2$.

We assume $\operatorname{char}(\mathbf{K})=0$. Here we want to show that if $H^{0}(X, L)$ induces a birational morphism (or, more generally, if for a general $T \in|L|, H^{0}(T, L \mid T)$ induces a birational morphism of $T$ ) then there is a lower bound for $g$ as a function of $p_{g}:=p_{g}(X)=p_{g}\left(X^{\prime}\right)$ which is of order $\left(p_{g}\right)^{2 / 3}$. The proof will show that there is also a lower bound of order $(c)^{2 / 3}$ for $g$ as a function of $c$. The example of the bundles $r \mathbf{O} X(1)$ on a degree $d$ smooth surface in $\mathbf{P}^{3}$ show that in general there is no such lower bound of order better than $\beta\left(p_{g}\right)^{2 / 3}$ with $\beta=(9 / 2)^{1 / 3}$.

Just to give a precise statement, we check the following result; however, note that the same proof (and in particular the part concerning $h^{0}(T, L \mid T)$ and [D]) can be used often to obtain better bounds; for the part concerning $h^{0}(T, L \mid T)$ see Remark 5.4.3.

Theorem 5.4.2. Assume char $(\mathbf{K})=0$. Assume E c-ample (any rank), $L$ spanned and that for a general $T \in|L|$ the morphism associated to $H^{0}(T, L \mid T)$ is 
birational; set $a:=h^{0}(T, L \mid T)$ (hence $\left.a \geq 3\right)$ and $\alpha^{\prime}:=\left((a-2)^{1 / 3}\right) / 2$. Set $c:=K_{X^{\prime}}^{2}$ and $p_{g}:=p_{g}(X)$. Then $g:=g(L) \geq \alpha^{\prime}\left(c-\left(c^{2 / 3}\right) / 2\right)^{2 / 3}$ and $g \geq(1 / 2) \alpha^{\prime}\left(p_{g}-\right.$ $\left.\left(p_{g}\right)^{2 / 3}\right)^{2 / 3}$. In particular, since $a \geq 3$, we have $\alpha^{\prime} \geq 1 / 2$.

Proof. If $\tau \geq\left((c)^{2 / 3}\right) / 2$; we conclude by Remark 5.4.1. Assume $\tau<\left((c)^{2 / 3}\right)$ $/ 2$. Note that $c \geq 2 p_{g}-4$ by [D] (and also $c \geq 2 p_{g}+2(q-4)$ with inequality if $X^{\prime}$ is not the product of two genus 2 curves), $c \geq 2 p_{g}$ if $q>0, c \geq 3 p_{g}-2 q+9$ if $\left|K_{X^{\prime}}\right|$ is composed with a pencil, $c \geq 3 p_{g}+q-7$ if $\left|K_{X^{\prime}}\right|$ is birational, $c \geq 2 p_{g}+4(q-4)$ if $\left|K_{X^{\prime}}\right|$ has degree two, and so on ([D]). Hence $K^{2} \geq 2 p_{g}-4-\left(p_{g}\right)^{2 / 3}$ and the bound with $c$ gives the bound with $p_{g}$. Set $d:=L^{2}$. Fix a general $T \in|L|$. Since $H^{0}(T, L|T|)$ induces a birational morphism on $T$, by Castelnuovo's bound on the genus we have $2(a-2) g \leq d^{2}$, hence $(a-2) K L \leq d^{2}$. Since $K^{2}>0$, and $d K^{2} \leq(K L)^{2}$ by Hodge index theorem, we get the inequality.

Remark 5.4.3. Here we assume $E$ ample and spanned and give better bounds on $a:=h^{0}(T, L|T|)$ (hence improve 5.4.2). Since $h^{0}(X, L) \leq 1+a$, and $E$ is spanned, to obtain $a \geq 4$ it is sufficient to exclude the case $\operatorname{rank}(E)=2$ and $h^{0}(X, L)=$ $h^{0}(X, E)+1=4$. This would give an extension

$$
0 \rightarrow L^{*} \rightarrow 30_{X} \rightarrow E \rightarrow 0
$$

(hence $c_{1}(E)^{2}=c_{2}(E)$, contradicting the ampleness of $E$; alternatively: by (11) $E$ is a pull back of $T \mathbf{P}^{2}(-1)$, which is not ample).

$\S 6$. In this short section we give a related result in a strictly positive characteristic situation: the case of uniruled surfaces of general type. This section was motivated by the form of the characteristic 0 lower bound for $g(L)$ given in [LR] (which is much better if $\chi\left(\mathbf{O}_{X}\right)<0$ ) and the proof by Sheperd-Barron ([SB2], th. 7) that surfaces of general type with $c_{2}<0$ are uniruled. In positive characteristic it seems to be an open question the existence of a surface $X$ of general type with $\chi\left(\mathbf{O}_{X}\right)<0$ (see [SB2], Remark at p. 268); by [SB2], th. 8, there is no such $X$ if $p>7$.

In this section we fix a prime $p$ and we assume $\operatorname{char}(\mathbf{K})=p$. Let $X$ be a surface of general type which is uniruled. We assume $\operatorname{Alb}(X) \neq 0$ (hence the image, $C$ of the Albanese map $\alpha: X \rightarrow \operatorname{Alb}(X)$ must be a curve and indeed it is easy to check that it is a smooth curve). Set $q:=p_{a}(C)$. To avoid empty statements, we will assume $q>1$, i.e. $\operatorname{dim}(\operatorname{Alb}(X))>1$. Let $E$ be a rank $r$ vector bundle on $X$; set $L:=\operatorname{det}(E)$. We have the following result.

Proposition 6.1. (i) Assume that $E$ is spanned by global sections outside finitely many points, that it is generically c-ample and that a general curve $D \in|L|$ is integral. We have:

(i) Assume $p \geq 5$. Set $w:=(p-1) / 2$; then $g(L) \geq r w q-w+1$. 
(ii) Assume $p=2$ or 3 . Then $g(L) \geq 2 r q-2 r+1$.

Proof. Let $T$ be a general fiber of $\alpha$. Let $\phi: T^{\prime} \rightarrow T$ be the normalization map; $T^{\prime}$ is rational. By assumption $E \mid T$ is spanned. For part (ii) we fix any singular point $P \in T$. For part (i) we fix a point $P \in T_{\text {sing }}$ at which the support of the sheaf $\phi_{*}\left(\mathbf{O}_{T^{\prime}}\right) / \mathbf{O}_{T}$ has length at least $(p-1) / 2$; the existence of such a point $P$ follows from the formal case of a theorem of Tate $([T])$. Fix $s \in H^{0}(T, E \mid T)$ with $s \neq 0, s(P)=0$. The existence of $s$ and the spannedness of $\phi^{*}((E \mid T))$ implies that $\phi^{*}((E \mid T))$ is a direct sum of $r$ line bundles of degree at least 2 in case (ii) and at least $w$ in case (i). Hence the result follows from Riemann-Hurwitz.

$\S 7$. Note that by [Gi], prop. 2.2, on a smooth curve of genus $q>0$ for all integers $r, d$ with $r>0$ and $d>0$ there are ample rank $r$ bundles of degree $d$. Here we will consider the possible inequalities between $c_{1}(E)^{2}$ and $c_{2}(E)$ when $E$ is a rank 2 ample and spanned vector bundle on a smooth surface $X$. It is a general fact (using only the ampleness of $E$ ) that $c_{1}(E)^{2}>c_{2}(E)([\mathrm{FL}])$; this is the only such inequality which is stable under pull-backs by all finite morphisms. However, usually the classification problems are not stable under finite pull-backs, and one is often very happy to have other inequalities on the Chern classes involved in the problem. For the case $c_{1}(E)^{2}-c_{2}(E)$ low, see [B2]. If $\operatorname{char}(\mathbf{K})=0$ in [B3] we proved (again only assuming the ampleness of $E$ ) that $c_{1}(E)^{2} \leq c_{2}(E)^{2}+2 c_{2}(E)+1$ (if $c_{2}(E)>1$ ) and that if $E$ is spanned and we have equality then $X \cong \mathbf{P}^{2}$ and $E \cong 2 \mathbf{O}(1)$. Here we will show that if we do not have equality and $E$ is spanned, then we have a much better inequality (see theorem 7.1). We stress this kind of jumps in the possible Chern classes. In particular it means that an inequality with a borderline example is not always "sharp in a very meaningful way".

Theorem 7.1. Assume $\operatorname{char}(\mathbf{K})=0$. Let $E$ be a rank 2 ample and spanned vector bundle on a smooth surface $X$. Then

$$
c_{1}(E)^{2} \leq c_{2}(E)^{2}+2 c_{2}(E)+1
$$

and if we have equality in (12), then $X \cong \mathbf{P}^{2}$ and $E \cong \mathbf{O}(1) \otimes \mathbf{O}(a)($ with $a+1=$ $\left.c_{1}(E)\right)$. Assume $c_{1}(E)^{2}>4 c_{2}(E)$ and that $(X, E) \neq\left(\mathbf{P}^{2}, \mathbf{O}(1) \otimes \mathbf{O}(a)\right)$. Then

$$
c_{1}(E)^{2} \leq\left(\left(c_{2}(E)^{2} / 2\right)+2 c_{2}(E)+2\right.
$$

and if we have equality in (12) then either $X$ is a smooth quadric $Q \subset \mathbf{P}^{3}$ and $E \cong \mathbf{O}(1) \otimes \mathbf{O}\left(c_{1}(E)-1\right)$, or $X$ is a double covering $u: X \rightarrow \mathbf{P}^{2}$ and $E$ has $u^{*}(\mathbf{O}(1))$ as quotient. If $(X, E)$ is not as above, then:

$$
c_{1}(E)^{2} \leq\left(\left(\left(c_{2}(E)-1\right)^{2}\right) / 2\right)+2 c_{2}(E)+1 .
$$


Proof. The first statement was just inserted for completeness and to show the hugeness of the jumps, since it was proved in [B3], th. 0.1 and Remark 1.2. Since $c_{1}(E)^{2}>4 c_{2}(E)$ by assumption, $E$ is not Bogomolov stable and, as in [R] we obtain an extension:

$$
0 \rightarrow N \rightarrow E \rightarrow M \otimes \mathbf{I}_{Z} \rightarrow 0
$$

with $Z \subset X, \operatorname{dim}(Z)=0, M$ and $N$ line bundles, $c_{1}(E)=M+N, c_{2}(E)=$ $M \cdot N+\operatorname{length}(Z)$ and (since $c_{1}(E)$ is ample) $(N+M)(N-M)>0$, i.e. $N^{2}>M^{2}$. Set $z:=\operatorname{length}(Z)$. First assume $z=0$ (hence $M$ is obviously ample and spanned); we have $M \cdot N=c_{2}(E)$. If $M^{2}=1, M$ induces an isomorphism of $X$ to $\mathbf{P}^{2}$. Assume $M^{2} \geq 2 ;$ since $N^{2} \geq M^{2}$, by Hodge index we have $N^{2} \leq\left(c_{2}(E)^{2}\right) / 2$ which gives (13); the proof gives also that if we have equality in (13), then $(X, E)$ is as claimed. If $M^{2} \geq 3$ (and $z=0$ ) we have an inequality stronger than (14). Now assume $z>0$; since $E$ is spanned, $M \otimes \mathbf{I}_{Z}$ is spanned and this implies $M^{2} \geq z$. We claim that $M^{2}>z$. Assume by constradiction $M^{2}=z$. Since $E$ is spanned there are two curves $A, B$ in $|M|$ with $A \cap B=Z$ as scheme. Using (15) it is easy to check that $E \mid A$ has a quotient line bundle of degree 0 , contradicting the ampleness of $E$. By the claim if $z>0$ we have $M^{2} \geq 2$ (while $M \cdot N=c_{2}(E)-z<c_{2}(E)$ ) and we conclude.

REMARK 7.2. The proof of 7.1 shows how (under the assumption $c_{1}(E)^{2}>$ $4 c_{2}(E)$ ) one can continue the analysis and improve (in many situations) the results.

REMARK 7.3. If $\operatorname{char}(\mathbf{K})=p>0$, by [SB1] the Reider type proof of 7.1 works if $\kappa(X) \leq 1$ (with the possible exception if $p=2$ or 3 when $\kappa(X)=1$ and $X$ is a quasi-elliptic fibration).

REMARK 7.4. The Reider type proof of 7.1 used the assumption $c_{1}(E)^{2}>$ $4 c_{2}(E)$ only to be sure that $E$ is Bogomolov unstable (and hence to obtain (15)). Hence the same result holds assuming the instability of $E$. We will discuss in the following remark the case of a stable (or semistable) bundle.

REMARK 7.5. Let $E$ be a rank 2 ample and spanned vector bundle on $X$. Set $L:=\operatorname{det}(E)$. Assume that $E$ is $L$-semistable (resp. $L$-stable) (in the sense of Mumford-Takemoto). This means that every filtration (15) of $E$ has $L \cdot N \leq L \cdot M$ (resp. $L \cdot N<L \cdot M$ ), i.e. $\tau:=M^{2}-N^{2} \geq 0$ (resp. $>0$ ); $\tau$ measures the degree of $L$-stability of $E$. Fix any such filtration (15) and set $z:=\operatorname{length}(Z)$. By the proof of the claim at the end of the proof of 7.1 we have $M^{2} \geq z+1$. Hence $c_{1}(E)^{2} \geq 2 c_{2}(E)+2-\tau$.

\section{References}

[ B1 ] E. Ballico, On globally generated vector bundles on surfaces with $c_{2}=1$, Geom. Dedicata, 27 (1988), 319-324. 
[ B2 ] E. Ballico, On ample and spanned vector bundles with zero $\Delta$-genera, Manuscripta Math., 70 (1990), 153-155.

[ B3 ] E. Ballico, On the Chern classes of ample vector bundles, Boll. Un. Mat. Ital., (7) 6-A (1992), 359-364.

[ B4 ] E. Ballico, Strange bundles on $\mathbf{P}^{2}$ and elementary transformations, Ann. Univ. Ferrara, 37 (1991), 1-11.

[BPV] W. Barth, C. Peters, A. van de Ven, Compact, complex surfaces, Ergebnisse der Math. 4, Springer-Verlag, 1984.

[ D ] O. Debarre (with an appendix by A. Beauville), Inégalités numériques pour les surfaces de type général, Bull. Soc. Math. France, 110 (1982), 319-346.

[ F ] T. Fujita, Ample vector bundles of small $c_{1}$-sectional genus, J. Math. Kyoto Univ., 29 (1989), 1-16.

[ FL ] W. Fulton, R. Lazarsfeld, The numerical positivity of ample vector bundles, Ann. of Math., 118 (1983), 35-60.

[ Gi ] D. Gieseker, p-ample bundles and their Chern classes, Nagoya Math. J., 43 (1971), 91-116.

[ H1 ] R. Hartshorne, Ample vector bundles, Publ. I.H.E.S., 29 (1966), 63-94.

[ H2 ] R. Hartshorne, Ample vector bundles on curves, Nagoya Math. J., 43 (1971), 73-89.

[ LR ] A. Lanteri, F. Russo, A lower bound for sectional genera of ample and spanned vector bundles on algebraic surfaces, preprint.

[ $\mathrm{Ma}$ ] M. Maruyama, Elementary transformations of algebraic vector bundles, in: Algebraic Geometry-Proceedings, La Rabida, pp. 241-266, Lect. Notes in Math. 961, SpringerVerlag, 1983.

[ N ] M. Nagata, On self-intersection number of a section on a ruled surface, Nagoya Math. J., 37 (1970), 191-196.

[ $\mathrm{O}$ ] T. Oda, Vector bundles on an elliptic curve, Nagoya Math J., 43 (1971), 41-72.

[ R ] I. Reider, Vector bundles of rank 2 and linear systems on algebraic surfaces, Ann. of Math., 127 (1988), 309-316.

[ $\mathrm{Ru}$ ] F. Russo, Some inequalities for ample and spanned vector bundles on algebraic surfaces, preprint.

[ SB1 ] N. Sheperd-Barron, Unstable vector bundles and linear systems on surfaces in characteristic $p$, Invent. Math., 106 (1991), 243-262.

[ SB2 ] N. Sheperd-Barron, Geography for surfaces of general type in positive characteristic, Invent. Math., 106 (1991), 263-274.

[ T ] J. Tate, Genus change in inseparable extensions of function fields, Proc. Amer. Math. Soc., 3 (1952), 400-404. 